\title{
Curtailing the cost of anesthetic drugs: prudent economics or an infringement of clinical autonomy?
}

\author{
Alan F. Merry, MBChB $\cdot$ Richard Hamblin, BA
}

Received: 8 June 2015/Accepted: 16 July 2015/Published online: 4 August 2015

(c) Canadian Anesthesiologists' Society 2015

In this issue of the Journal, Drs Tabing, Ehrenfeld, and Wanderer report statistically significant savings in the cost of anesthetic drugs following an intervention that in essence rendered the use of remifentanil, dexmedetomidine, and desflurane available only by request. ${ }^{1}$ The majority of the gains were attributable to reductions in the use of remifentanil and desflurane-dexmedetomidine was used in only a small number of cases either before or after the intervention.

Is this an example of prudent custodianship of financial resources that all institutions should emulate, or is it an infringement of the right and duty of clinicians to provide optimal care to every patient?

For many high-income countries, the costs of healthcare have escalated steadily over recent decades. Estimates for 2012 place the proportion of gross domestic product (GDP) allocated for healthcare expenditure at $16.9 \%$ for the USA, $10.9 \%$ for Canada, and a median of $9.2 \%$ for countries in the Organisation for Economic Co-operation and Development. ${ }^{2}$ These figures compare with $10.4 \%, 8.1 \%$, and $7.0 \%$, respectively, allocated 30 years ago. ${ }^{3}$ There is ample evidence that health outcomes are substantially influenced by indirect determinants of healthcare $e^{4,5}$ and that increasing

A. F. Merry, MBChB ( $\square)$

Department of Anaesthesiology, University of Auckland, Private Bag 92019, Auckland 1142, New Zealand

e-mail: a.merry@auckland.ac.nz

\section{A. F. Merry, MBChB}

Green Lane Department of Anaesthesia, Auckland City Hospital, Auckland, New Zealand

R. Hamblin, BA

Health Quality and Safety Commission, Wellington,

New Zealand direct expenditure on healthcare (above a reasonable threshold) does not necessarily result in improved health outcomes. $^{6}$

Resources are limited, so increasing the proportion of GDP spent on healthcare reduces the proportion available for other important investments into infrastructure (e.g., housing and road construction), other essential services (e.g., education and defence), and social support, all of which impact on the health of the population.

Not all clinicians accept that they should balance their ethical imperative to provide optimal care to each individual under their care with a wider duty to the entire population of their country. For those who don't accept this principle, the question arises as to how they will be able to continue to provide optimal care to each and every future patient if and when the money runs out. For those who do accept this view, the question shifts to whether or not the benefit a patient receives from a particular treatment or drug (in this case, desflurane or remifentanil) is sufficient to justify the additional cost over possible alternatives (isoflurane or sufentanil).

In relation to the first question, the intervention in this study did not restrict the choice of agents. It simply made it slightly more difficult to use three expensive drugs, and thereby, it presumably increased awareness of their cost and ensured that clinicians wanting to use them actually made an active decision to do so. This can hardly be construed as an unreasonable challenge to clinical autonomy.

In relation to the second question, the changes in drug usage do not appear to have had any important impact on the quality of patient care. They were not associated with an increase in the frequency of prolonged time to extubation, unplanned intraoperative intubations, or re-intubations in the postanesthesia care unit. Postoperative nausea and vomiting decreased a little, and the use of naloxone increased a little. Other patient outcomes were not assessed, but overall, it 
seems unlikely that any serious harm ensued. Indeed, if one considers the relative importance of ensuring universal access to essential healthcare affordably, the outcomes that really matter should be measured. For example, prolonging emergence from an anesthetic by a minute or two would arguably be of little relevance.

Were the savings worthwhile? These authors estimated a reduction in cost of $\$ 10.95$ per case, translating to between $\$ 233,857.57$ and $\$ 359,411.85$ per year. To place this in context, the authors estimated that the savings could fund an extra 2.17 nurse anesthetists or 1.02 attending physicians working full time in an institution with 274 anesthesia providers in total. As discussed in the paper, the drugs represent only a proportion of the total cost of anesthesia and an even smaller proportion of the total cost of clinical care, including anesthesia, surgery, and hospitalization. Tabing et al. cite work from Michigan in which $24 \%$ of a department of anesthesiology's budget went towards anesthetic drugs and 54\% went towards salaries. ${ }^{7}$ They also cite research suggesting that intraoperative anesthesia costs account for $5.6 \%$ of total perioperative costs, with labour accounting for most of the costs. ${ }^{8}$ In itself, then, it does seem easy to argue that saving $\$ 10$ or so per case is hardly worth the trouble involved in this initiative.

We think that such a conclusion would miss the real message of this paper: this research has shown that a relatively simple intervention can change anesthesia practice and save money without any important downside.

To understand the full potential implication of this outcome one needs to consider the question of variation in healthcare. This variation is substantial. ${ }^{9-11}$ Variation is deemed appropriate if it reflects the differing needs or values of individual patients with differing comorbidities and preferences. Instead, much of the variation in healthcare reflects differences in beliefs and approaches between practitioners and institutions rather than differences between patients. This variation is harder to justify.

Fisher and Wennberg ${ }^{12}$ identify three categories of medical services. Effective care should be received by all patients. Arguably, if there is evidence to support the superiority of a particular drug in a particular setting, all practitioners should use that drug in such settings whether or not it is more expensive (within reason) than less effective alternatives. Preference-sensitive care is typically seen when the value of treatments (including drugs) is uncertain. Objectively, if there is no evidence to support one regimen over another, patient preference should be considered, and if there is no such preference, the simplest and least expensive option should be used. Supply-sensitive care includes newly introduced drugs and technology, amongst other things. Adoption often precedes clear evidence of value. ${ }^{13}$ Supplysensitive care is often associated with overutilization. In a sense, by placing a modest impediment to "supply", Tabing et al. have indirectly shone a light on an element of anesthesia practice that might well fall into this category. Their intervention may, in effect, be seen as having produced a small shift in the direction of reducing practitioner-centric variation in anesthetic practice.

Reduction of variation, often thought of as standardization, has long been recognized in other industries (such as aviation) as an important element in improving the reliability and safety of complex processes (anesthesia is a complex process). Indeed, Tabing et al. point out that reducing the number of drugs presented to anesthesia providers might well reduce the potential for medication error. It seems to us that the case is strong for standardization of the indications for particular drugs and techniques, at least within each institution. This does not imply the "dumbing down" of clinical practice-it is rather about increasing the emphasis on making choices that truly matter.

The intervention described by Tabing et al. could be seen as just a first step. The potential for saving could be considerable if all practitioners within an institution could be persuaded to agree to reduce the range of drugs and techniques they use in most routine cases to a small number of agents-taking cost into account and articulating agreed indications for more expensive drugs or approaches. Tabing et al. have shown that simply making access to certain drugs more difficult is sufficient to reduce their use. For institutions or departments of anesthesia, this points the way towards implementing a more general consensus on the restriction of all expensive agents to situations in which they are truly justified.

The increasing effectiveness of healthcare in general and drugs in particular has come with progressively increasing costs. The need to maximize the value of healthcare expenditure applies both to institutions competing in the marketplace and to nations grappling with the challenge of providing universal healthcare affordably. In high-income settings, it seems reasonable to argue that no patient should be denied a better drug simply on the basis that it is somewhat more expensive, provided its advantages are supported by evidence. In the absence of such evidence, the extra expense is wasteful and represents an opportunity cost that, in the big picture, threatens the sustainability of high-quality services.

How much evidence can reasonably be expected in this context? Clinically important outcome data from large randomized trials will often be absent-indeed, if this level of evidence were present, substantial variation in the use of that drug would be unlikely. We think, as a minimum, there should be a formalized consensus amongst the clinicians within any given institution that worthwhile advantages can be articulated for the more expensive agent. Collective support for routine use of any one drug does not preclude 
individual practitioners from exercising discretion when specific reasons exist to justify this alternative-notably patient factors or preferences. The small barrier introduced by Tabing et al. seems to be a remarkably reasonable way to operationalize a consensus-based approach to the optimal use of all anesthesia drugs within any particular department. This sort of move from practitioner-centric variation to a more standardized practice of anesthesia in which variation is patient-centric may well be acceptable to practitioners in both categories described above. It may also be the key towards maximizing the value obtained from limited resources and thereby ensuring that more of the expensive drugs and therapies of proven value are indeed available for those patients who really need them.

The importance of standardization on the basis of evidence applies as much to surgical and other aspects of healthcare as it does to anesthesia. Tabing et al. have shone a light on a deceptively important aspect of anesthesia practice with potentially far-reaching implications. Anesthesiologists have an opportunity to provide leadership in redefining the way cost-effective choices are made-collectively and rationally-in the provision of healthcare.

\section{Contenir les coûts des médicaments anesthésiques: une mesure d'économie prudente ou une atteinte à l'autonomie clinique?}

\begin{abstract}
Dans ce numéro du Journal, les Drs Tabing, Ehrenfeld et Wanderer rapportent avoir obtenu des économies statistiquement significatives sur le coût total des médicaments anesthésiques, après l'introduction de mesures rendant certains agents disponibles sur demande spéciale seulement: le rémifentanil, la dexmédétomidine et le desflurane. ${ }^{1}$ La majorité des économies furent attribuables à des réductions dans l'utilisation du rémifentanil et du desflurane, la dexmédétomidine n'ayant été utilisée que dans un nombre restreint de cas avant et après l'introduction de ces mesures.
\end{abstract}

S'agit-il là d'un exemple de tutelle prudente des ressources financières à imiter par toutes les institutions, ou plutôt d'une atteinte au droit et au devoir du clinicien, de prodiguer des soins optimaux à chaque patient?

Dans de nombreux pays à revenus élevés, les coûts des soins de santé ont augmenté de façon constante au fil des dernières décennies. On estime qu'en 2012, la part du produit national brut (PNB) allouée aux soins de santé était de $16,9 \%$ aux États-Unis, $10,9 \%$ au Canada et, en moyenne (médiane), de 9,2 \% dans les pays de l'Organisation de coopération et de développement économiques $(\mathrm{OCDE}) .^{2}$ En comparaison, ces chiffres étaient de $10,4 \%, 8,1 \%$ et $7,0 \%$, respectivement, il y a 30 ans. $^{3}$ Nombreuses sont les données probantes faisant état de l'impact substantiel de facteurs indirects sur les résultats cliniques en soins de santé ${ }^{4,5}$ tout comme celles attestant qu'en augmentant les dépenses directes en santé (au-delà d'un seuil raisonnable), on n'améliore pas forcément les résultats cliniques. ${ }^{6}$

Les ressources sont limitées; par conséquent, en augmentant la part du PNB attribuée à la santé, on réduit les fonds disponibles pour d'autres investissements importants, comme par exemple dans les infrastructures (par ex., la construction de maisons et de routes), dans d'autres services essentiels (par ex. l'éducation et la défense) ainsi que dans les programmes sociaux - des domaines ayant tous un impact sur la santé de la population.

Ce ne sont pas tous les cliniciens qui acceptent de contrebalancer leur propre impératif déontologique - offrir des soins optimaux à chaque personne confiée à leurs soins - avec leur devoir plus large envers la population de leur pays. Pour ceux qui s'opposent à ce principe, la question se pose, à savoir comment ils pourront continuer, à l'avenir, à offrir des soins optimaux à chacun de leurs patients, si et quand il n'y aura plus d'argent pour le faire. Pour ceux qui approuvent ce principe, en revanche, la question est de savoir si les bénéfices qu'un patient retire d'un traitement ou d'un médicament en particulier (dans le cas présenté dans ce numéro, le desflurane ou le rémifentanil) justifient ou non le coût supplémentaire par rapport aux autres alternatives possibles (l'isoflurane ou le sufentanil).

En ce qui touche à la première question, les mesures décrites dans cette étude ne restreignaient aucunement le choix des agents anesthésiques. Elles rendaient simplement l'utilisation de trois médicaments dispendieux un peu plus difficile, augmentant ainsi, supposément, la prise de conscience quant à leur coût et garantissant que les cliniciens désirant les utiliser réfléchissent activement avant de prendre leur décision. On peut difficilement interpréter cette mesure comme une atteinte déraisonnable à l'autonomie clinique.

Quant à la deuxième question, les changements en matière d'utilisation des médicaments ne semblent pas avoir d'impact important sur la qualité des soins aux patients. Ces modifications n'ont pas été associées à une augmentation de la fréquence de délais d'extubation, d'intubations peropératoires imprévues, ou de réintubations en salle de réveil. Les nausées et vomissements postopératoires ont un peu diminué, et l'utilisation de naloxone a légèrement augmenté. Bien qu'aucun autre impact sur les patients ne fût évalué, il semble peu probable qu'une complication grave ne 
soit survenue. En fait, si l'on tient compte de l'importance relative de garantir un accès universel aux soins de santé essentiels à un prix raisonnable, il faudrait mesurer d'autres résultats importants. Le fait de retarder le réveil d'une minute ou deux après une anesthésie ne devrait pas être très important.

Les économies en ont-elle valu la peine? Les auteurs ont estimé une économie de 10,95 \$ par cas, ce qui équivaut à un montant allant de 233857,57 \$̀̀ 359411,85 \$ par an. Pour mettre ces chiffres en contexte, les auteurs ont estimé que l'économie pourrait financer 2,17 infirmières anesthésistes de plus, ou 1,02 médecin à temps plein dans une institution comptant au total 274 anesthésiologistes et intervenants en soins critiques. Comme le mentionne l'article, les médicaments ne représentent qu'une partie du coût total de l'anesthésie, et une part encore plus petite du coût total des soins cliniques, y compris l'anesthésie, la chirurgie et l'hospitalisation. Tabing et coll. citent des données provenant du Michigan, dans lesquels $24 \%$ du budget d'un département d'anesthésiologie étaient consacrés aux médicaments anesthésiques et $54 \%$ aux salaires. ${ }^{7}$ Ils citent également des recherches suggérant que les coûts peropératoires de l'anesthésie ne forment que 5,6\% des coûts périopératoires totaux, la main d'œuvre étant responsable de la plus grande partie de ces coûts. ${ }^{8}$ En soi, il semble donc facile de dire qu'une économie de 10 \$ par cas ne vaut pas vraiment les inconvénients occasionnés par cette initiative.

Selon nous, une telle conclusion passerait à côté du message véritable de cet article: ces travaux démontrent qu'une mesure relativement simple peut changer la pratique de l'anesthésie et économiser de l'argent sans inconvénient majeur.

Afin de comprendre toutes les implications potentielles de tels résultats, il convient de penser à la question des variations dans la pratique des soins de santé. Les variations décrites ici sont de taille. ${ }^{9-11}$ Une variation est acceptable si elle répond à des besoins ou valeurs spécifiques d'un patient ayant des comorbidités ou des préférences particulières. Au lieu de cela, la vaste majorité des variations que l'on observe en santé reflètent des différences de croyances ou d'approches entre les praticiens et les institutions, plutôt que des différences entre les patients. Ce type de variations est plus difficile à justifier.

Fisher et Wennberg ${ }^{12}$ identifient trois catégories de services médicaux. Les soins efficaces sont les soins qui devraient être reçus par tous les patients. Il ne fait aucun doute que si des données probantes appuient la supériorité d'un médicament en particulier dans un contexte en particulier, alors tous les praticiens devraient utiliser ce médicament dans de tels contextes, qu'il soit plus dispendieux (dans la mesure du raisonnable) que des alternatives moins efficaces ou non. Les soins selon la préférence sont en général prodigués lorsque la valeur d'un traitement (y compris d'un médicament) est incertaine. D'un point de vue objectif, si aucune donnée n'appuie un régime posologique plutôt qu'un autre, il convient alors de tenir compte des préférences du patient et, s'il n'a pas de préférence, d'utiliser l'option la plus simple et la moins dispendieuse. Les soins sensibles à l'approvisionnement comprennent, entre autres, les médicaments et technologies ajoutés récemment. L'adoption d'un traitement donné précède souvent des données probantes claires attestant son efficacité. ${ }^{13}$ Les soins sensibles à l'approvisionnement sont souvent sur-utilisés. D'une certaine façon, en entravant quelque peu «l'approvisionnement», Tabing et coll. ont indirectement mis le doigt sur un aspect de la pratique de l'anesthésie qui pourrait tomber dans cette catégorie. En fait, leur intervention peut être perçue comme ayant provoqué une légère réorientation vers une réduction des variations liées au praticien dans la pratique de l'anesthésie.

Dans d'autres secteurs d'activités (tels que l'aviation), la réduction de la variation, qu'on assimile souvent à une standardisation, est depuis longtemps une mesure reconnue pour améliorer la fiabilité et la sécurité des processus complexes (l'anesthésie est aussi un processus complexe). En effet, Tabing et coll. soulignent que la réduction du nombre de médicaments proposés aux anesthésiologistes pourrait bien entraîner une réduction du potentiel d'erreurs médicamenteuses. Selon nous, l'idée de normaliser les indications de médicaments et de techniques en particulier, du moins au sein de chaque institution, est bonne. Cela ne veut pas dire « automatiser » la pratique clinique; plutôt, il s'agit de mettre plus d'emphase sur les choix qui comptent vraiment.

L'application de mesures telles que celles décrites par Tabing et coll. dans leur étude pourrait être perçue comme un premier pas. Le potentiel d'économies pourrait être considérable si l'on pouvait convaincre tous les praticiens au sein d'une même institution de réduire l'éventail de médicaments et de techniques utilisés dans les cas de routine à un nombre restreint d'agents - en tenant compte de leur coût et en créant des indications acceptées pour les médicaments et les approches à coût plus élevé. Tabing et coll. ont démontré qu'il suffisait de rendre l'accès à certains médicaments plus difficile pour réduire leur utilisation. Pour les institutions et les départements d'anesthésie, cette étude montre la voie vers la création d'un consensus plus répandu concernant la restriction de tous les agents dispendieux aux situations qui les justifient véritablement.

L'efficacité croissante des soins de santé en général et des médicaments en particulier s'est accompagnée de coûts eux aussi croissants. Le besoin d'optimiser la valeur des dépenses en santé s'applique tant au niveau des institutions qui rivalisent sur le marché, qu'au niveau des nations aux prises avec le défi de procurer des soins de santé universels à un prix raisonnable. Lorsque les moyens le permettent, il 
est tout à fait raisonnable de s'assurer que chaque patient ait accès au meilleur médicament possible, peu importe son prix - pourvu que l'utilisation de ce médicament soit appuyée par des données probantes. En l'absence de telles données, les coûts supplémentaires sont du gaspillage et représentent un coût d'opportunité qui, globalement, met en péril la durabilité des services de haute qualité.

Combien de données probantes peut-on raisonnablement obtenir dans un tel contexte? Des données cliniques importantes tirées d'études randomisées d'envergure feront souvent défaut - en effet, si l'on disposait de ce type de données, il n'y aurait pas une si grande variation dans l'utilisation des médicaments. Selon nous, il devrait au minimum y avoir un consensus formel parmi les cliniciens au sein d'une même institution, à l'effet qu'un agent plus dispendieux possède véritablement des avantages de taille. Un consensus collectif concernant l'utilisation de routine d'un agent donné n'empêche pas chaque praticien d'utiliser son jugement lorsqu'il y a des raisons spécifiques qui justifient l'utilisation d'un autre agent - notamment des facteurs ou des préférences de patient. Le petit obstacle introduit par Tabing et coll. semble constituer une façon efficace de mettre en œuvre une approche fondée sur le consensus, quant à l'utilisation optimale de tous les médicaments anesthésiques, dans quelque département que ce soit. Ce type de réorientation, d'une variation de pratique centrée sur le praticien vers une pratique plus normalisée de l'anesthésie, dans laquelle les variations dans la pratique sont centrées sur le patient, pourrait être acceptable pour les praticiens des deux catégories décrites ci-dessus. Ce pourrait également être la clé d'une optimisation de la valeur obtenue pour nos ressources limitées, et ainsi garantir qu'une plus grande quantité des médicaments et de traitements dispendieux, ayant fait leurs preuves, soient disponibles pour les patients qui en ont véritablement besoin.

L'importance de la normalisation dans la pratique clinique, fondée sur les données probantes, s'applique autant à la chirurgie et aux autres aspects des soins de santé qu'à l'anesthésie. Tabing et coll. ont mis en lumière un aspect important et méconnu de la pratique anesthésique, ayant des implications d'une envergure potentiellement considérable. Les anesthésiologistes ont la possibilité de devenir des chefs de file en redéfinissant la façon dont les choix rentables sont faits - de façon collective et rationnelle - dans l'octroi des soins de santé.
Conflicts of interest Dr. Alan Merry is Chair of the Health Quality and Safety Commission. He owns approximately 10\% shares in Safer Sleep LLC, which produces an anesthesia safety and recording system.

Conflit d'intérêt Le Dr Alan Merry est président de la Commission de qualité et sécurité des soins de santé néozélandaise (Health Quality and Safety Commission). Il possède approximativement $10 \%$ des parts de Safer Sleep LLC, qui fabrique un système de sécurité et d'enregistrement anesthésique.

\section{References}

1. Tabing AK, Ehrenfeld JM, Wanderer JP. Limiting the accessibility of cost-prohibitive drugs reduces overall anesthetic drug costs: a retrospective before and after analysis. Can J Anesth 2015; 62: this issue. DOI:10.1007/s12630-015-0442-8.

2. OECD Health spending (indicator) 2015; DOI:10.1787/8643 de7e-en (accessed June 2015).

3. OECD Health Statistics 2014 - Frequently Requested Data. Available from URL: http://www.oecd.org/els/health-systems/ oecd-health-statistics-2014-frequently-requested-data.htm (accessed June 2015).

4. Marmot M. The Status Syndrome: How Social Standing Affects Our Health and Longevity. Times Books; 2004.

5. Deaton A. Policy implications of the gradient of health and wealth. Health Aff (Millwood) 2002; 21: 13-30.

6. Davis K, Stremikis K, Squires D, Schoen C. Mirror, Mirror on the Wall: How the Performance of the U.S. Health Care System Compares Internationally - 2014 Update. Available from URL: http://www.commonwealthfund.org/ /media/files/publications/ fund-report/2014/jun/1755_davis_mirror_mirror_2014.pdf (accessed June 2015).

7. Szocik JF, Learned DW. Impact of a cost containment program on the use of volatile anesthetics and neuromuscular blocking drugs. J Clin Anesth 1994; 6: 378-82.

8. Macario A, Vitez TS, Dunn B, McDonald T. Where are the costs in perioperative care? Analysis of hospital costs and charges for inpatient surgical care. Anesthesiology 1995; 83: 1138-44.

9. McGlynn E, Asch S, Adams J, et al. The quality of health care delivered to adults in the United States. N Engl J Med 2003; 348: 2635-45.

10. Wennberg JE, Peters $P G J r$. Unwarranted variations in the quality of health care: can the law help medicine provide a remedy/remedies? Spec Law Dig Health Care Law 2004; 305: 925.

11. Fisher ES, Wennberg DE, Stukel TA, Gottlieb DJ, Lucas FL, Pinder EL. The implications of regional variations in Medicare spending. Part 2: health outcomes and satisfaction with care. Ann Intern Med 2003; 138: 288-98.

12. Fisher ES, Wennberg JE. Health care quality, geographic variations, and the challenge of supply-sensitive care. Perspect Biol Med 2003; 46: 69-79.

13. Van Brabandt $H$, Neyt $M$, Hulstaert $F$. Transcatheter aortic valve implantation (TAVI): risky and costly. BMJ 2012; 345: e4710. 